TAMKANG JOURNAL OF MATHEMATICS

Volume 33, Number 2, Summer 2002

\title{
A NEW OSTROWSKI TYPE INEQUALITY INVOLVING INTEGRAL MEANS OVER END INTERVALS
}

\author{
P. CERONE
}

\begin{abstract}
The Ostrowski inequality expresses bounds on the deviation of a function from its integral mean. The current article obtains bounds for the deviation of a function from a combination of integral means over the end intervals covering the entire interval. Perturbed expressions are also determined via the Chebychev functional. A variety of earlier results are recaptured as particular instances of the current development.
\end{abstract}

\section{Introduction}

Let the functional $S(f ; a, b)$ be defined by

$$
S(f ; a, b)=f(x)-\mathcal{M}(f ; a, b),
$$

where

$$
\mathcal{M}(f ; a, b)=\frac{1}{b-a} \int_{a}^{b} f(x) d x .
$$

The functional $S(f ; a, b)$ represents the deviation of $f(x)$ from its integral mean over $[a, b]$.

In 1938, A. Ostrowski proved the following integral inequality [11].

Theorem 1. Let $f:[a, b] \rightarrow \mathbb{R}$ be continuous on $[a, b]$ and differentiable on $(a, b)$ and assume $\left|f^{\prime}(x)\right| \leq M$ for all $x \in(a, b)$. Then the inequality

$$
|S(f ; a, b)| \leq\left[\left(\frac{b-a}{2}\right)^{2}+\left(x-\frac{a+b}{2}\right)^{2}\right] \frac{M}{b-a}
$$

holds for all $x \in[a, b]$. The constant $\frac{1}{4}$ is best possible.

In a series of papers, Dragomir and Wang [4] - [7] proved (1.3) and other variants for $f^{\prime} \in L_{p}[a, b]$ for $p \geq 1$, the Lebesgue norms making use of a Peano kernel approach and

Received April 13, 2001; revised May 17, 2001.

2000 Mathematics Subject Classification. Primary 26D15, 26D20; Secondary 65Xxx

Key words and phrases. Ostrowski inequality, Chebychev functional. 
Montgomery's identity [10, p. 585]. Montgomery's identity states that for absolutely continuous mappings $f:[a, b] \rightarrow \mathbb{R}$

$$
f(x)=\frac{1}{b-a} \int_{a}^{b} f(t) d t+\frac{1}{b-a} \int_{a}^{b} p(x, t) f^{\prime}(t) d t,
$$

where the kernel $p:[a, b]^{2} \rightarrow \mathbb{R}$ is given by

$$
p(x, t)= \begin{cases}t-a, & a \leq t \leq x \leq b, \\ t-b, & a \leq x \leq t \leq b .\end{cases}
$$

If we assume that $f^{\prime} \in L_{\infty}[a, b]$ and $\left\|f^{\prime}\right\|_{\infty}:=e s s \sup _{t \in[a, b]}\left|f^{\prime}(t)\right|$ then $M$ in (1.3) may be replaced by $\left\|f^{\prime}\right\|_{\infty}$.

Dragomir and Wang [4] - [7] utilising an integration by parts argument, ostensibly Montgomery's identity (1.4), obtained

$$
\begin{aligned}
& |S(f ; a, b)| \\
& \leq \begin{cases}{\left[\left(\frac{b-a}{2}\right)^{2}+\left(x-\frac{a+b}{2}\right)^{2}\right] \frac{\left\|f^{\prime}\right\|_{\infty}}{b-a},} & f^{\prime} \in L_{\infty}[a, b] ; \\
{\left[\frac{(x-a)^{q+1}+(b-x)^{q+1}}{q+1}\right]^{\frac{1}{q}} \frac{\left\|f^{\prime}\right\|_{p}}{b-a},} & f^{\prime} \in L_{p}[a, b], \quad p>1, \quad \frac{1}{p}+\frac{1}{q}=1 ; \\
{\left[\frac{b-a}{2}+\left|x-\frac{a+b}{2}\right|\right] \frac{\left\|f^{\prime}\right\|_{1}}{b-a},} & \end{cases}
\end{aligned}
$$

where $f:[a, b] \rightarrow \mathbb{R}$ is absolutely continuous on $[a, b]$ and the constants $\frac{1}{4}, \frac{1}{(q+1)^{\frac{1}{q}}}$ and $\frac{1}{2}$ respectively are sharp.

The current paper obtains bounds on the deviation of a function from integral means from the end of the interval that cover the whole interval. The Ostrowski type results are recaptured as special cases. Following an identity obtained in Section 2 and the resulting bounds, perturbed results arising from the Chebychev functional are investigated in Section 3. The final Section 4 applies the results to the cumulative distribution function.

\section{Some Results}

We commence with the following identity which although of interest in itself, it will be used to obtain bounds.

Lemma 1. Let $f:[a, b] \rightarrow \mathbb{R}$ be an absolutely continuous mapping. Denote by $P(x, \cdot):[a, b] \rightarrow \mathbb{R}$ the kernel given by

$$
P(x, t)= \begin{cases}\frac{\alpha}{\alpha+\beta}\left(\frac{t-a}{x-a}\right), & t \in[a, x] \\ \frac{-\beta}{\alpha+\beta}\left(\frac{b-t}{b-x}\right), & t \in(x, b]\end{cases}
$$


where $\alpha, \beta \in \mathbb{R}$ nonnegative and not both zero, then the identity

$$
\int_{a}^{b} P(x, t) f^{\prime}(t) d t=f(x)-\frac{1}{\alpha+\beta}\left[\frac{\alpha}{x-a} \int_{a}^{x} f(t) d t+\frac{\beta}{b-x} \int_{x}^{b} f(t) d t\right]
$$

holds.

Proof. From (2.1), we have

$$
\begin{aligned}
\int_{a}^{b} P(x, t) f^{\prime}(t) d t= & \frac{\alpha}{\alpha+\beta} \int_{a}^{x}\left(\frac{t-a}{x-a}\right) f^{\prime}(t) d t-\frac{\beta}{\alpha+\beta} \int_{x}^{b}\left(\frac{b-t}{b-x}\right) f^{\prime}(x) d t \\
= & \left.\frac{\alpha}{\alpha+\beta}\left\{\left(\frac{t-a}{x-a}\right) f(t)\right]_{t=a}^{x}-\frac{1}{x-a} \int_{a}^{x} f(t) d t\right\} \\
& \left.-\frac{\beta}{\alpha+\beta}\left\{\left(\frac{b-t}{b-x}\right) f(t)\right]_{t=x}^{b}-\frac{1}{b-x} \int_{x}^{b} f(t) d t\right\},
\end{aligned}
$$

where the integration by parts formula has been utilised on the separate intervals $[a, x]$ and $(x, b]$. Simplification of the expressions readily produces the identity as stated.

Theorem 2. Let $f:[a, b] \rightarrow \mathbb{R}$ be an absolutely continuous mapping and define

$$
\mathcal{T}(x ; \alpha, \beta):=f(x)-\frac{1}{\alpha+\beta}[\alpha \mathcal{M}(f ; a, x)+\beta \mathcal{M}(f ; x, b)],
$$

where $\mathcal{M}(f ; a, b)$ is the integral mean as defined by $(1,2)$, then

$$
\begin{aligned}
& |\mathcal{T}(x ; \alpha, \beta)| \\
& \leq \begin{cases}{[\alpha(x-a)+\beta(b-x)] \frac{\left\|f^{\prime}\right\|_{\infty}}{2(\alpha+\beta)},} & f^{\prime} \in L_{\infty}[a, b] ; \\
{\left[\alpha^{q}(x-a)+\beta^{q}(b-x)\right]^{\frac{1}{q}} \frac{\left\|f^{\prime}\right\|_{p}}{(q+1)^{\frac{1}{q}}(\alpha+\beta)},} & f^{\prime} \in L_{p}[a, b], p>1, \frac{1}{p}+\frac{1}{q}=1 ; \\
{\left[1+\frac{|\alpha+\beta|}{\alpha+\beta}\right] \frac{\left\|f^{\prime}\right\|_{1}}{2},} & \end{cases}
\end{aligned}
$$

where $\|h\|$. are the usual Lebesgue norms for $h \in L .[a, b]$ with

$$
\|h\|_{\infty}:=e s s \sup _{t \in[a, b]}|h(t)|<\infty
$$

and

$$
\|h\|_{p}:=\left(\int_{a}^{b}|h(t)|^{p} d t\right)^{\frac{1}{p}}, 1 \leq p \leq \infty
$$

Proof. Taking the modulus of (2.2) we have from (2.3) and (1.2)

$$
|\mathcal{T}(x ; \alpha, \beta)|=\left|\int_{a}^{b} P(x, t) f^{\prime}(t) d t\right| \leq \int_{a}^{b}|P(x, t)|\left|f^{\prime}(t)\right| d t
$$


where we have used the well known properties of the integral and modulus.

Thus, for $f^{\prime} \in L_{\infty}[a, b]$ from (2.5) gives

$$
|\mathcal{T}(x ; \alpha, \beta)| \leq\left\|f^{\prime}\right\|_{\infty} \int_{a}^{b}|P(x, t)| d t
$$

from which a simple calculation using (2.1) gives

$$
\begin{aligned}
\int_{a}^{b}|P(x, t)| d t & =\frac{\alpha}{\alpha+\beta} \int_{a}^{x} \frac{t-a}{x-a} d t+\frac{\beta}{\alpha+\beta} \int_{x}^{b} \frac{b-t}{b-x} d t \\
& =\left[\frac{\alpha}{\alpha+\beta}(x-a)+\frac{\beta}{\alpha+\beta}(b-x)\right] \int_{0}^{1} u d u
\end{aligned}
$$

and hence the first inequality results.

Further, using Hölder's integral inequality, we have for $f^{\prime} \in L_{p}[a, b]$ from (2.5)

$$
|\mathcal{T}(x ; \alpha, \beta)| \leq\left\|f^{\prime}\right\|_{p}\left(\int_{a}^{b}|P(x, t)|^{q} d t\right)^{\frac{1}{q}},
$$

where $\frac{1}{p}+\frac{1}{q}=1$ with $p>1$. Now

$$
\begin{aligned}
(\alpha+\beta)\left(\int_{a}^{b}|P(x, t)|^{q} d t\right)^{\frac{1}{q}} & =\left[\alpha^{q} \int_{a}^{x}\left(\frac{t-a}{x-a}\right)^{q} d t+\beta^{q} \int_{x}^{b}\left(\frac{b-t}{b-x}\right)^{q} d t\right]^{\frac{1}{q}} \\
& =\left[\alpha^{q}(x-a)+\beta^{q}(b-x)\right]^{\frac{1}{q}}\left(\int_{0}^{1} u^{q} d u\right)^{\frac{1}{q}}
\end{aligned}
$$

and so the second inequality is obtained.

Finally, for $f^{\prime} \in L_{1}[a, b]$ we have from (2.5) and using (2.1)

$$
|\mathcal{T}(x ; \alpha, \beta)| \leq \sup _{t \in[a, b]}|P(x, t)|\left\|f^{\prime}\right\|_{1},
$$

where

$$
(\alpha+\beta) \sup _{t \in[a, b]}|P(x, t)|=\max \{\alpha, \beta\}=\frac{\alpha+\beta}{2}+\left|\frac{\alpha-\beta}{2}\right|
$$

and so the theorem is now completely proven.

Remark 1. It should be noted that from (2.3) and (1.1),

$$
(\alpha+\beta) \mathcal{T}(x ; \alpha, \beta)=\alpha \mathcal{S}(f ; a, x)+\beta \mathcal{S}(f ; x, b)
$$

and so from (1.5) using the triangle inequality produces

$$
\begin{aligned}
& |(\alpha+\beta) \mathcal{T}(x ; \alpha, \beta)| \\
\leq & \left\{\begin{array}{l}
\frac{\alpha}{2}(x-a)\left\|f^{\prime}\right\|_{\infty,[a, x]}+\frac{\beta}{2}(b-x)\left\|f^{\prime}\right\|_{\infty,[x, b]}, \\
\alpha\left(\frac{x-a}{q+1}\right)^{\frac{1}{q}}\left\|f^{\prime}\right\|_{p,[a, x]}+\beta\left(\frac{b-x}{q+1}\right)^{\frac{1}{q}}\left\|f^{\prime}\right\|_{p,[x, b]}, \\
\alpha\left\|f^{\prime}\right\|_{1,[a, x]}+\beta\left\|f^{\prime}\right\|_{1,[x, b]},
\end{array}\right.
\end{aligned}
$$


where for $[c, d] \subseteq[a, b]$

$$
\|h\|_{p,[c, d]}:=\left(\int_{c}^{d}|h(t)|^{p} d t\right)^{\frac{1}{p}}, p \geq 1
$$

and $\|h\|_{\infty,[c, d]}:=e s s \sup _{t \in[c, d]}|h(t)|$.

That is,

$$
\begin{aligned}
& |(\alpha+\beta) \mathcal{T}(x ; \alpha, \beta)| \\
\leq & \left\{\begin{array}{l}
{[\alpha(x-a)+\beta(b-x)] \frac{\left\|f^{\prime}\right\|_{\infty}}{2},} \\
{\left[\begin{array}{l}
\left.\alpha\left(\frac{x-a}{q+1}\right)^{\frac{1}{q}}+\beta\left(\frac{b-x}{q+1}\right)^{\frac{1}{q}}\right]\left\|f^{\prime}\right\|_{p}, \\
(\alpha+\beta)\left\|f^{\prime}\right\|_{1},
\end{array}\right.}
\end{array} \quad f^{\prime} \in L_{\infty}[a, b], p>1, \frac{1}{p}+\frac{1}{q}=1 ;\right.
\end{aligned}
$$

where the expression (2.8) involving the $\|\cdot\|_{p}$ norm is coarser.

The results of (2.7) in which the norms are evaluated over the two subintervals, although finer, they do require more work.

Remark 2. It is possible to reduce the amount of work alluded to in Remark 1 since we may write

$$
\begin{aligned}
& \alpha \mathcal{M}(f ; a, x)+\beta \mathcal{M}(f ; x, b) \\
= & \alpha \mathcal{M}(f ; a, x)+\frac{\beta}{b-x}\left[\int_{a}^{b} f(u) d u-\int_{a}^{x} f(u) d u\right] \\
= & {\left[\alpha-\beta\left(\frac{x-a}{b-x}\right)\right] \mathcal{M}(f ; a, x)+\beta\left(\frac{b-a}{b-x}\right) \mathcal{M}(f ; a, b) } \\
= & {[\alpha+\beta-\beta \rho(x)] \mathcal{M}(f ; a, x)+\beta \rho(x) \mathcal{M}(f ; a, b), }
\end{aligned}
$$

where

$$
\rho(x)=\frac{b-a}{b-x} .
$$

Thus, from $(2.3), \mathcal{T}(x ; \alpha, \beta)$ may be written in the following equivalent form

$$
\mathcal{T}(x ; \alpha, \beta)=f(x)-\left[\left(1-\frac{\beta}{\alpha+\beta} \rho(x)\right) \mathcal{M}(f ; a, x)+\frac{\beta}{\alpha+\beta} \rho(x) \mathcal{M}(f ; a, b)\right]
$$

so that for fixed $[a, b], \mathcal{M}(f ; a, b)$ is also fixed.

The following uniform bounds are valid.

Corollary 1. Let the conditions of Theorem 2 hold. Then

$$
\begin{aligned}
& \left|f(x)-\frac{1}{2}[\mathcal{M}(f ; a, x)+\mathcal{M}(f ; x, b)]\right| \\
& \leq \begin{cases}\frac{(b-a)}{4}\left\|f^{\prime}\right\|_{\infty}, & f^{\prime} \in L_{\infty}[a, b] ; \\
\left(\frac{b-a}{q+1}\right)^{\frac{1}{q}} \cdot \frac{\left\|f^{\prime}\right\|_{p}}{2}, & f^{\prime} \in L_{p}[a, b], p>1, \frac{1}{p}+\frac{1}{q}=1 ; \\
\frac{\left\|f^{\prime}\right\|_{1}}{2} . & \end{cases}
\end{aligned}
$$


Proof. The result is readily obtained on allowing $\beta=\alpha$ in (2.4) so that the left hand side is $\mathcal{T}(x ; \alpha, \alpha)$ from $(2.3)$.

Corollary 2. Let the conditions of Theorem 2 hold. Then

$$
\begin{aligned}
& \left|f\left(\frac{a+b}{2}\right)-\frac{2}{(b-a)(\alpha+\beta)}\left[\alpha \int_{a}^{\frac{a+b}{2}} f(u) d u+\beta \int_{\frac{a+b}{2}}^{b} f(u) d u\right]\right| \\
& \leq \begin{cases}\frac{(b-a)}{4}\left\|f^{\prime}\right\|_{\infty}, & f^{\prime} \in L_{\infty}[a, b] ; \\
{\left[\alpha^{q}+\beta^{q}\right]^{\frac{1}{q}}\left(\frac{b-a}{2(q+1)}\right)^{\frac{1}{q}} \cdot \frac{\left\|f^{\prime}\right\|_{p}}{\alpha+\beta},} & f^{\prime} \in L_{p}[a, b], p>1, \frac{1}{p}+\frac{1}{q}=1 ; \\
{\left[1+\frac{|\alpha-\beta|}{\alpha+\beta}\right] \frac{\left\|f^{\prime}\right\|_{1}}{2} .} & \end{cases}
\end{aligned}
$$

Proof. Placing $x=\frac{a+b}{2}$ in (2.3) and (2.4) produces the results as stated in (2.12).

Corollary 3. If (2.11) is evaluated at the midpoint then

$$
\begin{aligned}
& \left|f\left(\frac{a+b}{2}\right)-\frac{1}{b-a} \int_{a}^{b} f(t) d t\right| \\
& \leq\left\{\begin{array}{l}
\frac{(b-a)}{4}\left\|f^{\prime}\right\|_{\infty}, \quad f^{\prime} \in L_{\infty}[a, b] ; \\
\left(\frac{b-a}{q+1}\right)^{\frac{1}{q}} \cdot \frac{\left\|f^{\prime}\right\|_{p}}{2}, \quad f^{\prime} \in L_{p}[a, b], p>1, \frac{1}{p}+\frac{1}{q}=1 ; \\
\frac{\left\|f^{\prime}\right\|_{1}}{2} .
\end{array}\right.
\end{aligned}
$$

which is in agreement with (1.5) when $x=\frac{a+b}{2}$. The above result could also be obtained by taking $\alpha=\beta$ in (2.12) or equivalently $\alpha=\beta$ and $x=\frac{a+b}{2}$ in (2.4).

\section{Perturbed Results}

Perturbed versions of the results of the previous section may be obtained by using Grüss type results involving the Chebychev functional

$$
T(f, g)=\mathfrak{M}(f g)-\mathfrak{M}(f) \mathfrak{M}(g)
$$

with $\mathfrak{M}(f)$ being the integral mean of $f$ over $[a, b]$, namely

$$
\mathfrak{M}(f)=\frac{1}{b-a} \int_{a}^{b} f(t) d t .
$$

For $f, g:[a, b] \rightarrow \mathbb{R}$ and integrable on $[a, b]$, as is their product, then

$$
\begin{array}{rlrl}
|T(f, g)| & \leq T^{\frac{1}{2}}(f, f) T^{\frac{1}{2}}(g, g), & \text { Dragomir [3] for } f, g \in L_{2}[a, b] \\
& \leq \frac{\Gamma-\gamma}{2} T^{\frac{1}{2}}(f, f), & & \text { Matić et al. [8] for } \gamma \leq g(t) \leq \Gamma, t \in[a, b], \\
& \leq \frac{(\Gamma-\gamma)(\Phi-\phi)}{4}, & & \text { Grüss(see [9, pp. 295-310]), } \phi \leq f \leq \Phi, t \in[a, b] .
\end{array}
$$


Dragomir [3] obtains numerous results if either $f, g$ or both are known, although the first inequality in (3.3) has a long history (see for example [9, pp. 295-310]. The inequalities in (3.3) when proceeding from top to bottom are on order of decreasing coarseness.

The following theorem is valid.

Theorem 3. Let $f:[a, b] \rightarrow \mathbb{R}$ be an absolutely continuous mapping and $\alpha \geq 0, \beta \geq$ $0, \alpha+\beta \neq 0$ then

$$
\begin{aligned}
\left|\mathcal{T}(x, \alpha, \beta)-(x-\gamma) \frac{S}{2}\right| & \leq(b-a) \kappa(x)\left[\frac{1}{b-a}\left\|f^{\prime}\right\|_{2}^{2}-S^{2}\right]^{\frac{1}{2}}, \quad f^{\prime} \in L_{2}[a, b] ; \\
& \leq(b-a) \kappa(x) \frac{\Gamma-\gamma}{2}, \quad \gamma<f^{\prime}(t)<\Gamma, t \in[a, b] \\
& \leq(b-a) \frac{\Gamma-\gamma}{4},
\end{aligned}
$$

where, $\Gamma(x, \alpha, \beta)$ is as given by (2.3) or equivalently (2.10),

$$
\begin{aligned}
\gamma & =\frac{\alpha a+\beta b}{\alpha+\beta}, \quad S=\frac{f(b)-f(a)}{b-a}, \\
\kappa^{2}(x) & =\frac{1}{3}\left[\left(\frac{\alpha}{\alpha+\beta}\right)^{2}(x-a)+\left(\frac{\beta}{\alpha+\beta}\right)^{2}(b-x)\right]-\left(\frac{x-\gamma}{2(b-a)}\right)^{2} .
\end{aligned}
$$

Proof. Associating $f(t)$ with $P(x, t)$ and $g(t)$ with $f^{\prime}(t)$ then from (2.1) and (3.1) we obtain

$$
T\left(P(x, \cdot), f^{\prime}(\cdot)\right)=\mathfrak{M}\left(P(x, \cdot), f^{\prime}(\cdot)\right)-\mathfrak{M}(P(x, \cdot)) \mathfrak{M}\left(f^{\prime}(\cdot)\right)
$$

and so, on using identity (2.2),

$$
(b-a) T\left(P(x, \cdot), f^{\prime}(\cdot)\right)=\tau(x, \alpha, \beta)-(b-a) \mathfrak{M}(P(x, \cdot)) S
$$

where $S$ is the secant slope of $f$ over $[a, b]$ as given in (3.5). Now, from (2.2),

$$
\begin{aligned}
(b-a) \mathfrak{M}(P(x, \cdot)) & =\int_{a}^{b} P(x, t) d t \\
& =\frac{\alpha}{\alpha+\beta} \int_{a}^{x} \frac{t-a}{x-a} d t-\frac{\beta}{\alpha+\beta} \int_{x}^{b} \frac{b-t}{b-x} d t \\
& =(x-\gamma) \int_{0}^{1} u d u
\end{aligned}
$$

and combining with (3.6) gives the left hand side of (3.4).

Now, for the bounds on (3.7) from $(3.3)$ we have to determine $T^{\frac{1}{2}}(P(x, \cdot), P(x, \cdot))$ and $\phi \leq P(x, \cdot) \leq \Phi$. Firstly, we note however that

$$
0 \leq T^{\frac{1}{2}}\left(f^{\prime}(\cdot), f^{\prime}(\cdot)\right)=\left[\mathfrak{M}\left(\left(f^{\prime}(\cdot)\right)^{2}\right)-\mathfrak{M}^{2}\left(f^{\prime}(\cdot)\right)\right]^{\frac{1}{2}}
$$




$$
\begin{aligned}
& =\left[\frac{1}{b-a} \int_{a}^{b}\left[f^{\prime}(t)\right]^{2} d t-\left(\frac{\int_{a}^{b} f^{\prime}(t) d t}{b-a}\right)^{2}\right]^{\frac{1}{2}} \\
& =\left[\frac{1}{b-a}\left\|f^{\prime}\right\|_{2}^{2}-S^{2}\right]^{\frac{1}{2}} \\
& \leq\left(\frac{\Gamma-\gamma}{2}\right), \quad \text { where } \gamma \leq f^{\prime}(t) \leq \Gamma, t \in[a, b] .
\end{aligned}
$$

Now from (2.1), the definition of $P(x, t)$, we have

$$
T(P(x, \cdot), P(x, \cdot))=\mathfrak{M}\left(P^{2}(x, \cdot)\right)-\mathfrak{M}^{2}(P(x, \cdot))
$$

where from (3.8),

$$
\mathfrak{M}(P(x, \cdot))=\frac{x-\gamma}{2(b-a)},
$$

and

$$
\begin{aligned}
\mathfrak{M}\left(P^{2}(x, \cdot)\right) & =\left(\frac{\alpha}{\alpha+\beta}\right)^{2} \int_{a}^{x}\left(\frac{t-a}{x-a}\right)^{2} d t+\left(\frac{\beta}{\alpha+\beta}\right)^{2} \int_{x}^{b}\left(\frac{b-t}{b-x}\right)^{2} d t \\
& =\left[\left(\frac{\alpha}{\alpha+\beta}\right)^{2}(x-a)+\left(\frac{\beta}{\alpha+\beta}\right)^{2}(b-x)\right] \int_{0}^{1} u^{2} d u .
\end{aligned}
$$

Thus, substituting the above results into (3.10) gives

$$
0 \leq \kappa(x)=T^{\frac{1}{2}}(P(x, \cdot), P(x, \cdot))
$$

which is given explicitly by (3.6). Combining (3.7), (3.11) and (3.9) give, from the first inequality in (3.3), the first inequality in (3.4). Also, utilising the inequality in (3.9) produces the second result in (3.4).

Further, it may be noticed from the definition of $P(x, t)$ in (2.1) that for $\alpha, \beta \geq 0$ and $\alpha$ and $\beta$ not zero at the same time give

$$
\Phi=\sup _{t \in[a, b]} P(x, t) \quad \text { and } \phi=\inf _{t \in[a, b]} P(x, t),
$$

giving $\Phi=\frac{\alpha}{\alpha+\beta}$ and $\phi=\frac{-\beta}{\alpha+\beta}$.

Hence, from (3.7) and the last inequality in (3.3) gives the final result in (3.4) and the theorem is now completely proved.

\section{An Application to the Cumulative Distribution Function}

Let $X$ be a random variable taking values in the finite interval $[a, b]$ with cumulative distribution function $F(x)=P_{r}(X \leq x)=\int_{a}^{x} f(u) d u$, where $f$ is a probability density function. The following theorem holds. 
Theorem 4. Let $X$ and $F$ be as above. Then

$$
\begin{aligned}
& |(\alpha(b-x)-\beta(x-a)) F(x)-(x-a)[(\alpha+\beta)(b-x) f(x)-\beta]| \\
\leq & \begin{cases}(b-x)(x-a)[\alpha(x-a)+\beta(b-x)] \cdot \frac{\left\|f^{\prime}\right\|_{\infty}}{2}, & f^{\prime} \in L_{\infty}[a, b] ; \\
(b-x)(x-a)\left[\alpha^{q}(x-a)+\beta^{q}(b-x)\right]^{\frac{1}{q}} \cdot \frac{\left\|f^{\prime}\right\|_{p}}{(q+1)^{\frac{1}{q}}}, & f^{\prime} \in L_{p}[a, b], p>1, \frac{1}{p}+\frac{1}{q}=1 ;( \\
(b-x)(x-a)[\alpha+\beta+|\alpha-\beta|] \cdot \frac{\left\|f^{\prime}\right\|_{1}}{2}, & f^{\prime} \in L_{1}[a, b] .\end{cases}
\end{aligned}
$$

Proof. The proof follows in a straightforward manner from (2.4) of Theorem 2.

Using (2.10) for $\mathcal{T}(x ; \alpha, \beta)$ and (2.11) we obtain on using the fact that $\int_{a}^{b} f(u) d u=1$

$$
\begin{aligned}
& (\alpha+\beta)(x-a)(b-x) \mathcal{T}(x ; \alpha, \beta) \\
= & (\alpha+\beta)(x-a)(b-x) f(x)-[\alpha(b-x)-\beta(x-a)] F(x)-\beta(x-a) .
\end{aligned}
$$

Thus,

$$
\frac{-(\alpha+\beta)(x-a)(b-x)}{\alpha(b-x)-\beta(x-a)} \mathcal{T}(x ; \alpha, \beta)=F(x)-(x-a)\left[\frac{(\alpha+\beta)(b-x) f(x)-\beta}{\alpha(b-x)-\beta(x-a)}\right]
$$

and so taking the modulus and using (2.4) gives the stated result.

Corollary 4. Let $X$ be a random variable, $F(x)$ to cumulative distribution function and $f(x)$ the probability density function. Then

$$
\begin{aligned}
& \left|\left(\frac{a+b}{2}-x\right) F(x)-(x-a)\left[(b-x) f(x)-\frac{1}{2}\right]\right| \\
\leq & \begin{cases}(b-x)(x-a)(b-a) \cdot \frac{\left\|f^{\prime}\right\|_{\infty}}{2}, & f^{\prime} \in L_{\infty}[a, b] ; \\
(b-x)(x-a)(b-a)^{\frac{1}{q}} \cdot \frac{\left\|f^{\prime}\right\|_{p}}{2(q+1)^{\frac{1}{q}}}, & f^{\prime} \in L_{p}[a, b], p>1, \frac{1}{p}+\frac{1}{q}=1 ; \\
(b-x)(x-a) \cdot \frac{\left\|f^{\prime}\right\|_{1}}{2}, & f^{\prime} \in L_{1}[a, b] .\end{cases}
\end{aligned}
$$

Remark 3. The above results allow the approximation of $F(x)$ in terms of $f(x)$. The approximation of $R(x)=1-F(x)$ could also be obtained by a simple substitution. $R(x)$ is of importance in reliability theory where $f(x)$ is the p.d.f. of failure.

Remark 4. We may take directly from (2.3) and (2.4) $\beta=0$, assuming that $\alpha \neq 0$, to give

$$
|F(x)-(x-a) f(x)| \leq \begin{cases}\frac{(x-a)^{2}}{2}\left\|f^{\prime}\right\|_{\infty}, & f^{\prime} \in L_{\infty}[a, b] ; \\ (x-a)^{1+\frac{1}{q}} \cdot \frac{\left\|f^{\prime}\right\|_{p}}{(q+1)^{\frac{1}{q}}}, & f^{\prime} \in L_{p}[a, b], p>1, \frac{1}{p}+\frac{1}{q}=1 ; \\ (x-a)\left\|f^{\prime}\right\|_{1}, & f^{\prime} \in L_{1}[a, b]\end{cases}
$$

which agrees with (1.5) for $|S(f ; a, x)|$. 
Remark 5. The perturbed results of Section 3 could also be applied here, however, this will not be pursued further.

Remark 6. We may replace $f$ and $F$ (see [1] for related results) in any of the equations (4.1) - (4.3) so that the bounds are in terms of $\left\|f^{\prime}\right\|_{p}, p \geq 1$. Further, we note that

$$
\left.\int_{a}^{b} F(u) d u=u F(u)\right]_{a}^{b}-\int_{a}^{b} x f(x) d x=b-E[X] .
$$

\section{Acknowledgement}

The work for this paper was done while the author was on sabbatical at La Trobe University, Bendigo.

\section{References}

[1] N. S. Barnett and S. S. Dragomir, An inequality of Ostrowski's for cumulative distribution functions, Kyungpook Math. J., (in press).

[2] P. Cerone and S. S. Dragomir, Three point quadrature rules involving, at most, a first derivative, RGMIA Res. Rep. Coll. 2(1999), Article 8, [ONLINE] http://rgmia.vu.edu.au/v2n4.html

[3] S. S. Dragomir, Better bounds in some Ostrowski-Grüss type inequalities, Tamkang J. Math., (submitted).

[4] S. S. Dragomir and S. Wang, A new inequality Ostrowski's type in $L_{1}$ norm and applications to some special means and some numerical quadrature rules, Tamkang J. of Math. 28(1997), 239-244.

[5] S. S. Dragomir and S. Wang, A new inequality Ostrowski's type in $L_{p}$ norm, Indian J. of Math. 40(1998), 299-304.

[6] S. S. Dragomir and S. Wang, An inequality Ostrowski-Grüss type and its applications to the estimation of error bounds for some special means and for some numerical quadrature rules, Computers Math. Applic. 33(1997), 15-22.

[7] S. S. Dragomir and S. Wang, Applications of Ostrowski's inequality to the estimation of error bounds for some special means and to some numerical quadrature rules, Appl. Math. Lett. 11(1998), 105-109.

[8] M. Matić, J. E. Pečarić and N. Ujević, On new estimation of the remainder in generalised Taylor's formula, Math. Ineq. Appl. 2(1999), 343-361.

[9] D. S. Mitrinović, J. E. Pečarić and A. M. Fink, Classical and New Inequalities in Analysis, Kluwer Academic Publishers, 1993.

[10] D. S. Mitrinović, J. E. Pečarić and A. M. Fink, Inequalities for Functions and Their Integrals and Derivatives, Kluwer Academic Publishers, 1994.

[11] A. Ostrowski, Uber die Absolutabweichung einer differentienbaren Funktionen von ihren Integralimittelwert, Comment. Math. Hel. 10(1938), 226-227.

School of communications and informatics, Victoria University of Technology, P.O. Box 14428, Melbourne City MC, Victoria 8001, Australia.

E-mail: pc@matilda.vu.edu.au

URL:http://sci.vu.edu.au/staff/peterc.html 